\title{
Complicações que acometem pacientes pós cirúrgicos: revisão integrativa
}

\author{
Complications affecting postoperative patients: integrative review
}

Jacqueline Trajano Alves Godinho', Thais Sousa Modesto²

\section{RESUMO}

Introdução: $O$ pós cirúrgico compreende 24 horas da cirúrgia até a alta. Inúmeras complicações podem ocorrer neste tempo e ao identificar as principais complicações possíveis a equipe hospitalar pode atuar diminuindo custos, tempo de internação, qualidade do serviço prestado. Objetivo: Identificar as complicações e e alternativas de prevenção e controle no pós cirúrgico. Material e Método: Para atender este objetivo foi realizada uma revisão integrativa com 47 trabalhos nas bases de dados Literatura Latino-Americana e do Caribe em Ciências da Saúde e Medical Literature Analysis and Retrieval System Online, utilizando as palavras-chave complições pós cirúrgicas, pós operatório, abordagens fisioterapêuticas. Resultados: Os fatores que causam complicações pós operatórias mais citados foram a idade avançada, a intensa manipulação cirúrgica, os efeitos da anestesia e a utilização de circulação extracorpórea. As complicações encontradas no pós-operatório de cirurgia cardíaca foram principalmente pulmonares, nas quais se destacaram hipoxemia, diminuição da complacência pulmonar, pneumonia e atelectasia. Conclusão: As complicações pós operatórias são muitas e envolvem diversos fatores, porém com a atuação do profissional de fisioterapia acelera-se o processo de recuperação, diminui o tempo de internação e embora sejam citadas diversas métodologias de ação ainda não é determinada a intervenção mais eficaz. Asim cada unidade hospitalar cria rotinas próprias de ações hospitalares pré e pós-operatórias.

Palavras-chave: período pós operatório, cuidados pós operatórios, complicações pós operatórios.

\section{ABSTRACT}

Introduction: The surgical post comprises 24 hours from surgery to discharge. Numerous complications can occur at this time and when identifying the main complications possible, hospital staff can act to reduce costs, length of stay, quality of service provided. Objective: To identify the complications and alternatives of prevention and control in the postoperative period. Materials and Methods: In order to meet this objective, an integrative review was carried out with 47 papers in the Latin American and Caribbean Literature in Health Sciences and Medical Literature Analysis and Retrieval System Online databases, using the keywords postoperative complications, post operative, physiotherapeutic approaches. Results: The most frequent causes of postoperative complications were advanced age, intense surgical manipulation, the effects of anesthesia and the use of extracorporeal circulation. The complications found in the postoperative period of cardiac surgery were mainly pulmonary, in which hypoxemia, decreased pulmonary complacency, pneumonia and atelectasis were noted. Conclusion: The postoperative complications are many and involve several factors, but with the performance of the physiotherapy professional accelerates the recovery process, reduces the length of hospitalization and although several methods of action are cited, the most effective intervention is not yet determined. Thus, each hospital unit creates its own routines for pre and postoperative hospital actions.

Keywords: postoperative period, postoperative care, postoperative complications. 


\section{INTRODUÇAOO}

O estudo das complicações pós operatórias têm especial relevância por sua frequência, porque em parte são atribuíveis a deficiências na atenção à saúde, pelo impacto considerável sobre a saúde dos pacientes, pela repercussão econômica no gasto social e sanitário e por constituir um instrumento de avaliação da qualidade da assistência'.

O paciente com bom estado de saúde tolera bem uma intervenção cirúrgica. O acompanhamento rotineiro pós cirúrgico: do estado de consciência, das condições de hidratação e balanço hídrico, do estado das condições de ventilação e oxigenação, das condições hemodinâmicas, observam-se 0 estado das cicatrizes cirúrgicas e o funcionamento de drenos, sondas e cateteres. Durante a recuperação anestésica é dedicado cuidado especial ao estado hemodinâmico e às condições ventilatórias. $\mathrm{O}$ reinício da movimentação deve ser precoce, contudo depende do tipo e da extensão da cirurgia. Recomenda-se a mudança de decúbito várias vezes ao dia no sentido de prevenir o acúmulo de secreções e a atelectasia pulmonar².

O correto acompanhamento do dia a dia do paciente juntamente com uma intervenção fisioterapêutica corretamente prescrita durante o pós-operatório proporciona numerosos benefícios aos pacientes, reduzindo significantemente o número de complicações nesse período ${ }^{3}$.

Uma importante complicação pós operatória é a Síndrome do Imobilismo, que é um conjunto de alterações que ocorre no indivíduo acamado por período prolongado, sendo elas: redução da capacidade funcional dos sistemas - osteomuscular, tecido conjuntivo, tecido articular, sistema respiratório, sistema metabólico e sistema geniturinário. Ocorre perda de $5 \%$ a $6 \%$ de massa muscular por dia, e por volta de quatro semanas cerca de $50 \%$ da força inicial pode estar comprometida 4 .

Diante disso, o objetivo deste trabalho é identificar as complicações pós cirúrgicas e suas medidas de prevenção de modo a permitir que a equipe possa atuar melhorando a qualidade do trabalho realizado. Além de possibilitar a ampliação de trabalhos científicos na área tendo em vista a escassez de publicações pertinente sobre o assunto pertinente.

\section{MATERIAIS E METODOS}

A pesquisa caracteriza-se como revisão integrativa realizada no Portal de Pesquisa da Biblioteca Virtual em Saúde, encontrando como principais complicações pós cirúrgicas: 
síndrome da alça aferente, fístula anastomótica, perda de células endoteliais da córnea, síndrome do roubo coronário-subclávio, recuperação demorada da anestesia, síndrome pós-laminectomia, oclusão de enxerto vascular, hérnia incisional, hipertermia maligna, dor pós-operatória, síndrome pós-colecistectomia, síndromes pós-gastrectomia, hemorragia pós-operatória, náusea e vômito pós-operatório, síndrome pós-pericardiotomia, falha de prótese, infecções relacionadas à prótese, traumatismo por reperfusão, choque cirúrgico, síndrome do intestino curto, deiscência da ferida operatória, infecção da ferida operatória, vasoplegia. A partir dessa lista foi feita a busca para identicar pesquisas científicas que citassem suas ocorrências e relatasse os problemas referentes ao caso. Todos os trabalhos científicos buscados nas bases de dados nacionais e internacionais. Encontrando-se na Literatura Latino-Americana e do Caribe em Ciências da Saúde LILACS; Medical Literature Analysis and Retrieval System Online - MEDLINE.

Os critérios de inclusão foram: texto completo disponível, assunto principal complicações pós-operatórias, relacionar-se a humanos, país de afiliação Brasil, idioma português, publicadas entre 2000 a 2016 ou extemporâneas, porém relevantes. Já os critérios de exclusão, foram publicações de outros idiomas que não o português e de caráter pago. Os descritores utilizados foram: Fisioterapia; Terapia Intensiva; Unidade de Terapia Intensiva. O período de coleta de dados foi em julho de 2016 a 20 de janeiro de 2017. Após a seleção do material, leitura e fichamento dos dados, os mesmos foram analisados e discutidos visando ofertar uma noção maior sobre as principais complicações pós cirúrgicas e perceber a importância do fisioterapeuta no pós cirúrgico.

\section{DESENVOLVIMENTO}

\section{PRINCIPAIS COMPLICAÇÕES PÓS-OPERATÓRIAS}

Com base na literatura pesquisada é possível identificar como principais complicações pós-cirúrgicas as seguintes: síndrome da alça aferente, fístula anastomótica, perda de células endoteliais da córnea, síndrome do roubo coronáriosubclávio, recuperação demorada da anestesia, síndrome pós-laminectomia, oclusão de enxerto vascular, hérnia incisional, hipertermia maligna, dor pós-operatória, síndrome póscolecistectomia, síndromes pós-gastrectomia, hemorragia pós-operatória, náusea e vômito pós-operatório, síndrome pós-pericardiotomia, falha de prótese, infecções 
relacionadas à prótese, traumatismo por reperfusão, choque cirúrgico, síndrome do intestino curto, deiscência da ferida operatória, infecção da ferida operatória, vasoplegia.

\section{Síndrome da alça aferente}

É uma complicação incomum encontrada após gastrectomia. Apresentando-se de forma aguda no pós-operatório precoce, levando a um quadro de dor abdominal em andar superior, vômitos não biliosos e rápida deterioração clínica requerendo cirúrgia. A forma crônica manifesta-se por obstrução parcial (podendo ser completa), com história de dor epigástrica e naúseas, cerca de trinta minutos após as refeições, quando a pressão se eleva na alça aferente de seu conteúdo é descarregado dentro do estômago, produzindo vômitos biliosos sem restos alimentares ${ }^{5}$.

\section{Fístula anastomótica}

Complicação decorrente de procedimento bariátrico ocorrendo de 0 a $41 \%$ dos casos e causando cerca de $90 \%$ dos óbitos pós operatórios ${ }^{6}$.

\section{Perda de células endoteliais da córnea}

Ocorre principalmente após a cirurgia de catarata. Sabe-se que atualmente há cerca de 20 milhões de pessoas com catarata no mundo, e esta complicação pode afetar 1 a $2 \%$ das cirurgias de catarata 7 .

\section{Síndrome do roubo coronário-subclávio}

É definido como o fluxo sanguíneo invertido de uma artéria coronária, por meio de enxerto de artéria mamária interna em direção à subclávia médio-distal, e ocorre devido à estenose significativa ou oclusão total da porção proximal desta última. É uma causa rara, mas significante, de isquemia cardíaca após cirurgia de revascularização miocárdica e pode originar uma síndrome de mesmo nome e com manifestações típicas ${ }^{8}$. 


\section{Recuperação demorada da anestesia}

A recuperação da anestesia ocorre geralmente de maneira tranquila, eventualmente, podem ocorrer complicações. Observa-se que as condições clínicas pré-operatórias, a extensão e o tipo de cirurgia, a técnica e a duração da anestesia, as intercorrência cirúrgicas e/ou anestésicas, em geral, estão relacionadas com a frequência de complicações no período pós-operatório imediato. Entre a complicações que decorrem cita-se: náusea e vômito, suporte para patência de via aérea superior e hipotensão. Sendo importante observar: integridade das funções respiratória, cardiovascular e neuromuscular, o nível de consciência, a temperatura, a presença de sangramentos, dor, náuseas e vômitos e o débito urinário ${ }^{9}$.

\section{Síndrome pós-laminectomia}

A patologia vertebral que mais frequentemente motiva uma intervenção cirúrgica é a hérnia discal, canal lombar estenótico e a espondiloistese. Com a realização de cirurgia pode ocorrer a síndrome que é uma condição que acomete indivíduos previamente submetidos a cirurgia vertebral lombar e caracteriza-se pela persistência de um quadro doloroso de localização lombar e/ou com irradiação para os membros inferiores ${ }^{10}$.

\section{Oclusão de enxerto vascular}

É um fator de morbimortalidade cardiovascular ocorrendo 17 novos casos por 100.000 habitantes/ano. Relaciona-se à embolização ou à trombose arterial. Com o aumento da utilização de técnicas endovasculares no tratamento desse grupo de pacientes, devemos considerar a hiperplasia intimal, que pode ser responsável pela trombose do stent no diagnóstico diferencial. A utilização de trombolíticos pode não ser a melhor opção terapêutica nesses $\operatorname{casos}^{11}$.

\section{Hérnia incisional}

Considera-se aberturas da parede abdominal, como também as hérnias de disco vertebral, as meningoceles, as hérnias do pulmão através da parede torácica e outras. Alguns órgãos da cavidade abdominal podem sair da mesma, através de um ponto fraco da parede sendo conservada a integridade do peritônio e da pele ${ }^{12}$. 


\section{Hipertermia maligna}

É uma afecção hereditária e latente, caracterizada classicamente por uma síndrome hipermetabólica em resposta à exposição aos anestésicos voláteis (halotano, enflurano, isoflurano, sevoflurano e desflurano) e/ou succinilcolina. Incidindo a cada 50 mil anestesias realizadas em adultos e a cada 15 mil anestesias aplicadas a crianças. Em qualquer idade, qualquer grupo racial, sendo ambos os sexos suscetíveis ${ }^{13}$.

\section{Dor pós-operatória}

A maioria dos pacientes no pós-operatório das cirurgias de grande porte, como as cirurgias abdominais altas, cardíacas e pulmonares, queixam-se de dor, sendo esta responsável pela respiração superficial, com conseqüentemente diminuição da capacidade vital, capacidade residual funcional, retenção de secreção e atelectasia. Estas alterações precisam ser minimizadas o mais precocemente possível, tanto pela conduta analgésica adequada, quanto pela intervenção fisioterapêutica respiratória ${ }^{14}$.

\section{Síndrome pós-colecistectomia}

A colecistectomia videolaparoscópica é o método de escolha no tratamento da colecistite aguda ou crônica. Contudo, cerca de $10 \%$ a $50 \%$ dos pacientes persistem ou desenvolvem novos sintomas após o procedimento. Sendo os mesmos na maioria dos casos leves e inespecíficos, consistindo em náusea transitória, eructação, flatulência e indigestão. A causa mais comum de síndrome pós-colecistectomia são desordens extrabiliares, tais como pancreatite crônica, doença ulcerosa péptica, doença do refluxo gastroesofagiano e síndrome do intestino irritável. As principais causas da síndrome póscolecistectomia de origem biliar são: 1) estenose biliar, 2) cálculo de via biliar principal, 3) síndrome do coto do ducto cístico, 4) estenose ou discinesia do esfíncter de Oddi. A remoção parcial da vesícula biliar (vesícula residual ou vesícula remanescente) é uma causa rara desta síndrome ${ }^{15}$. 


\section{Síndromes pós-gastrectomia}

As síndromes pós-gastrectomias incluem a gastrite e esofagite de refluxo, a diarreia pós-vagotomia, as síndromes das alças aferente e eferente, e a Síndrome de dumping, cuja incidência pode atingir até $58 \%$ dos pacientes no pós-operatório de gastrectomias $^{16}$.

\section{Hemorragia pós-operatória}

Quando ocorre pode oferecer risco de vida ao paciente. Têm incidência de $10 \%$ e é considerada a complicação mais grave. Podendo ocorrer de forma primária (<24 horas) ou secundária ( $>24$ horas). Sendo a primária muito mais frequente que a secundária, sendo que este tipo de evento ocorre entre 6 a 8 horas de pós operatório na maioria das vezes $(76-89 \% \text { dos casos })^{17}$.

\section{Náusea e vômito pós-operatório}

As complicaões como febre, naúseas, vômitos, inabilidade para ingerir alimentos sólidos ou líquidos, otalgia, dor. Podendo atingir grande magnitude que ocasionem desidratação e necessidade de reposição volêmica ${ }^{17}$.

\section{Síndrome pós-pericardiotomia}

A síndrome pode ocorrer após qualquer procedimento terapêutico, particularmente cirúrgico que cause violação do pericárdio. $O$ intervalo de tempo entre o seu aparecimento e o evento clínico com o qual se correlaciona pode ser de quatro dias a um ano. Constituise de processo inflamatório preuro-pericârdico e do parêquima pulmonar, é habitualmente benigno e autolimitado ${ }^{18}$.

\section{Falha de prótese}

As falhas podem ser desencadeadas por vários fatores, como na seleção do material, erros de projeto, na produção e na colocação do implante; falhas de reparação do osso; ou ainda pela combinação desses fatores ${ }^{19}$. 


\section{Infecções relacionadas à prótese}

As infecções hospitalares são as mais freqüentes e importantes complicações ocorridas em pacientes hospitalizados. No Brasil, estima-se que 5\% a 15\% dos pacientes internados contraem alguma infecção hospitalar. Uma infecção hospitalar acresce, em média, 5 a 10 dias ao período de internação. Além disso, os gastos relacionados a procedimentos diagnósticos e terapêuticas da infecção hospitalar fazem com que o custo seja elevado. Recomenda-se 0 uso de antissépticos na higienização preventiva. Administração de antimicrobianos por um período de 48 horas. Embora tenha um risco de baixa ocorrência quando ocorre pode ter consequências potencialmente desastrosa ${ }^{20}$.

\section{Traumatismo por reperfusão}

É um evento inevitável no transplante de rim, tendo como consequências retardo na função do enxerto, aumento no tempo de hospitalização e dos custos, aumento no risco de rejeição aguda e potencial impacto negativo na evolução a longo prazo. Vários fatores estão implicados na fisiopatologia da lesão de isquemia e reperfusão, podendo ser divididos em dois paradigmas: hemodinâmico e imunológico. O hemodinâmico é classicamente descrito como a privação de oxigênio pela interrupção do fluxo sanguíneo, envolvendo diversos sistemas hormonais e pela produção de radicais livres de oxigênio após a reperfusão. O imunológico tem sido descrito mais recentemente e envolve as células do sistema imune, sobretudo as células $T$, como papel fundamental na lesão. Novas estratégias de prevenção dos impactos da lesão de isquemia e reperfusão têm sido estudadas, especialmente formas mais fisiológicas de preservação do órgão, como a preservação em máquina de perfusão e o uso de anticorpos depletores de linfócitos antes da reperfusão ${ }^{21}$.

\section{Choque cirúrgico}

O choque cirúrgico é devido a combinações, em várias proporções, de hemorragia externa, sangramento para dentro de tecidos lesionados e desidratação ${ }^{22}$. 


\section{Síndrome do intestino curto}

A retirada de uma ou mais porções do intestino delgado resulta em processos carenciais de maior ou menor gravidade, dependendo da extensão e do tempo de duração da doença, originando a desnutrição, má evolução clínica e aumento da morbidade e mortalidade. Portanto, a gravidade da desnutrição e das complicações metabólicas dependem da doença de base e fatores como o comprimento do intestino remanescente, ressecção da válvula íleo-cecal e/ou cólon, tempo decorrente da ressecção, presença de outras doenças sistêmicas, e idade do paciente ${ }^{23}$.

\section{Deiscência da ferida operatória}

Consiste na abertura espontânea de suturas cirúrgicas. É separação de planos profundos, seguida pela descarga de líquidos e subsequente hérnia incisional. Sua incidência pode variar cerca de 5,55\% nas cirurgias combinadas que por sua vez, podem ocorrer por infecção ou pelo excesso de tensão daquele tecido, isquemia e fatores que interfiram na cicatrização ${ }^{24}$.

\section{Infecção da ferida operatória}

É a complicação mais frequente do paciente operado e, em sua essência, pode ser classificada como hospitalar, por decorrer de cirurgias ou de procedimentos invasivos intra-hospitalares e está associada a diferentes níveis de gravidade, desde o acometimento do local da incisão até coleções intracavitárias e infecções relacionadas a próteses, aumentando o risco de septicemia e reabordagem cirúrgica. Este tipo de infecção apresenta morbidade significativa que prolonga o tempo de internação, aumenta o risco de readmissão hospitalar, admissão em unidade de terapia intensiva e óbito ${ }^{25}$.

\section{Vasoplegia}

Caracteriza-se por uma combinação de hipotensão, débito cardíaco normal ou aumentado e por uma redução da resistência sistêmica, sendo muitas vezes refratária ao uso de aminas vasoativas. Os pacientes aumentam sua morbidade e mortalidade, necessitando de fluidos e drogas vasoativas no período peri-operatório e pós-operatório ${ }^{26}$. 
PRINCIPAIS MEDIDAS FISIOTERÁPICAS PARA EVITAR COMPLICAÇÕES PÓSOPERATÓRIAS

Cada intervenção cirúrgica possui ações fisioterápicas próprias. Em uma situação ideal, a reabilitação começa com a educação do paciente antes da cirurgia e continua após a cirurgia com a intervenção direta de um fisioterapeuta seguida pelo autocuidado em longo praia. A consulta prévia deve avaliar a dor, amplitude de movimento e integridade articular, integridade da pele, desempenho muscular (força e resistência), postura, análise da marcha, estado funcional. Elabora-se então plano de tratamento com as precauções pré e pós operatórias, avaliação da mobilidade no leito e transferências, e plano de exercícios pós-operatórios iniciais, treino de marcha e cuidados com a ferida ${ }^{27}$.

Já no plano pós cirúrgico dar-se ênfase na minimização da dor, na prevenção de complicações pós-operatórias e na retenção de um grau seguro de mobilidade funcional, ao mesmo tempo protegendo o local cirúrgico. Posteriormente, à medida que os tecidos cicatrizam e o paciente se recupera da cirurgia, as intenções são dirigidas para a restauração ou melhora da amplitude de movimento, força, controle neuromuscular, estabilidade, equilíbrio, resistência muscular e cardiopulmonar, assim como para a habilidade do paciente de realizar todas as atividades funcionais necessárias e desejadas ${ }^{27}$.

\section{CONSIDERAÇOES FINAIS}

As UTIs concentram em seus leitos pacientes que com o passar do tempo podem vir apresentar um quadro mais agravante, melhora com benefícios ou traumas para o resto da vida. Por isso a perspectiva de ter um profissional qualificado, equipado com todas as tecnologias disponíveis faz com que o cidadão/cliente possa ter uma recuperação mais rápida e menos onerosa ao estado.

Faz-se necessário implementar protocolos que priorizem 0 tratamento fisioterapêutico pré-operatório, talvez a principal deficiência verificada. É imprescindível nesse período, além da anamnese cuidadosa, a instituição de momentos educativos onde se possa preparar o paciente para o período crítico do pós-operatório, quer seja por meio de palestras ou mesmo informações à beira do leito.

O planejamento e racionalização da atividade evitam improvisações, na medida em que definem com antecedência os agentes que serão envolvidos, propiciando-lhes treinar 
suas ações, desta forma eliminando ou minimizando os erros. É reconhecida a importância da conduta fisioterapêutica com exercícios respiratórios específicos no pósoperatório para reduzir o risco de complicações pulmonares, obtendo a melhora da ventilação pulmonar, diminuição da dor, aceleração do processo de recuperação pulmonar e reduzir o tempo de permanência do paciente no hospital.

\section{REFERENCIAS}

1. MOURA, Maria de Lourdes de Oliveira. Avaliação de eventos adversos cirúrgicos em hospitais do Rio de Janeiro, 2010.

2. DA SILVA STRACIERI, Luís Donizeti. Cuidados e complicações pós-operatórias. Medicina (Ribeirao Preto. Online), v. 41, n. 4, p. 465-468, 2008.

3. DE MELO, Antonio Quaresma; SILVA, Marcos Maciel Soares; MAIA, Marcelo Sousa; BRITO, Maria Andréia. Intervenção fisioterapêutica no pós-operatório de revascularização do miocárdio: uma perspectiva bibliográfica. Jornada de Iniciação Científica.

4. ALMEIDA, Cristiane Isabela de; SAAD, Marcelo; VIEIRA, Mario Sergio Rossi. Reabilitação em reumatologia geriátrica. Einstein, 2008.

5. PEREIRA, Gerson Alves; FÉRES, Omar; ANDRADE, José Ivan de; CENEVIVA, Reginaldo. Síndrome da alça aferente com necrose simulando pseudocisto de pâncreas. Rev. Col. Bras. Cir, v. 25, n. 2, p. 13840, 1998.

6. LOPES, Fábio; REIS, André Fonseca dos; CÔRTES, Bruno Juste Werneck; FABRINI, Dreyfus Silva; LIMA, Henrique Ribeiro; ROCHA, Luiz Carlos Gomes. Esofagojejunostomia após gastrectomia total: anastomose mecânica ou manual?. Rev. Col. Bras. Cir, v. 35, n. 5, p. 298-303, 2008.

7. GONÇALVES, Eliana Domingues; CAMPOS, Mauro; PARIS, Fabiana; GOMES, José Àlvaro Pereira; FARIAS, Charles Costa de. Ceratopatia bolhosa: etiopatogênese e tratamento. Arquivos Brasileiros de Oftalmologia, 2008.

8. MIILLER, Jean Carlo; CANDEMIL, Patrick Cardoso; LOURES, João Marcelo Gonçalves da Rocha; ORTIZ, Marco Rodrigo; GARCIA, Rogério Sanchez. Síndrome do roubo coronário-subclávio: relato de caso e revisão de literatura. J Vasc Bras, v. 11, n. 2, p. 166-70, 2012.

9. AMARAL, José Luiz Gomes do; FALCÃO, Luiz Fernando dos Reis. Recuperação pós-anestésica. In: Guia de anestesiologia e medicina intensiva. Editora Manole, 1르 edição, pg 776. 2011.

10.GASPAR, Ana Teresa; MORGADO, Sandra; ANTUNES, Filipe. Dor após cirurgia lombar: imagem e reflexos. Revista da Sociedade Portuguesa de Medicina Física e de Reabilitação, v. 21, n. 1, 2012.

11.ROSSI, Fabio Henrique; UEHARA, Milton Kiyonory; CHEN, Juliana, MAIA, Thiago Emilio Burza; DAROLD, Eduardo Mulinari, MARTINS, Andréia Silveira; IZUKAWA, Nilo Mitsuro; PRAKASAN, Akash Kuzhiparambil. Oclusão arterial aguda de stent fêmoro-poplíteo. Jornal Vascular Brasileiro, v. 8, n. 3, p. 267-270, 2009.

12. LEX, Ary. Hérnias em geral: revisão didática. Revista de Medicina, v. 47, n. 1, p. 13-38, 1963.

13. AMARAL, J. L. G.; Carvalho RB, Cunha LBP, Batti MAS, Issy AM, Habib AK, Silva HCA, Cagnolatti DC, Simões CM. Hipertermia maligna. Associação Médica Brasileira e Conselho Federal de Medicina (orgs). Projeto Diretrizes. São Paulo: AMB/CFM, 2009. 
DOI 10.18606/2318-1419/amazonia.sci.health.v7n2p2-13
Godinho JTA, Modesto TS.

Complicações que acometem pacientes pós cirúrgicos: revisão integrativa

14.XAVIER, Thaiza Teixeira; TORRES, Gilson de Vasconcelos; ROCHA, Vera Maria da. Dor pós-operatória: características quanti-qualitativa relacionadas a toracotomia póstero-lateral e esternotomia. Acta Cir Bras, v. 20, n. 1, p. 63-8, 2005.

15. COELHO, Júlio CU; NASSIF, Aissar E.; CAMPOS, Antonio Carlos L. Residual gallbladder. Revista do Colégio Brasileiro de Cirurgiões, v. 29, n. 6, p. 367-369, 2002.

16. SILVA, Alcino Lázaro da; GOMES, Célio Geraldo de Oliveira. Assessment of the gastro-jejuno-duodenal transit after jejunal pouch interposition. ABCD. Arquivos Brasileiros de Cirurgia Digestiva (São Paulo), v. 28, n. 4, p. 231-233, 2015.

17. WIIKMANN, Vivian; PRADO, Flávio Augusto Passarelli; CANIELLO, Marcello; DI FRANCESCO, Renata C; MIZIARA, Ivan D. Complicações pós operatórias em tonsilectomias. Rev Bras Otorrinolaringología, v. 70, n. 4, p. 446-51, 2004.

18.MENDES, Ana; SILVA, Susana Lopes da; FONSECA, Marina; FERREIRA, Carlos; SANTOS, J. Meneses. Sindroma pós-pericardiotomia-Caso clínico. Medicina Interna, vol. 8, n. 4, 2001.

19. WILLIAMS, David F. A review of metallurgical failure modes in orthopedic implants. In: Proc. Int. Symp. on Retrieval and Analysis of Orthopaedic Implants. 1977. p. 11-21.

20. MACHADO, A. Ferraz AAB, Ferraz E, Arruda E, Nobre J, Konkewicz LR, Pimentel ML, Leão MTC, Trabasso P, Grimbaum R. Prevenção da infecção hospitalar. Projeto Diretrizes, p. 16-17, 2001.

21. REQUIÃO-MOURA, Lúcio Roberto; DURÃO JUNIOR, Marcelino de Souza; MATOS, Ana Cristina Carvalho de; PACHECO-SILVA, Alvaro. Lesão de isquemia e reperfusão no transplante renal: paradigmas hemodinâmico e imunológico. Einstein (16794508), v. 13, n. 1, 2015.

22. HAMMER, Gary D.; MCPHEE, Stephen J. Fisiopatologia da Doença. McGraw Hill Brasil, 2015.

23.NONINO, Carla Barbosa; BORGES, Ricardo Martins; PASQUALI, Luciana Sicca; MARCHINI, Júlio Sérgio. Terapia nutricional oral em $p$ terapia nutricional oral em pacientes com síndrome do intestino curto cientes com síndrome do intestino curto. Rev. Nutr, v. 14, n. 3, p. 201-205, 2001.

24.SILVA, Bianka Sousa Martins; LUA, Iracema; DAS MERCES, Magno Conceição; ANDRADE, Luana Machado; OLIVEIRA, Daniela Sousa. Cuidados de enfermagem na assistência domiciliar a um paciente com deiscência abdominal. Biológicas \& Saúde, v. 4, n. 15, 2014.

25.BELLUSSE, Gislaine Cristhina; RIBEIRO, Julio Cesar; CAMPOS, Fabrício Ribeiro de; POVEDA, Vanessa de Brito; GALVÃO, Cristina Maria. Fatores de risco de infecção da ferida operatória em neurocirurgia. Acta paul. enferm, v. 28, n. 1, p. 66-73, 2015.

26. MORAIS, Vinícius Daudt. Agentes Vasopressores em Sindrome Vasoplégica após Cirurgia Cardíaca: Revisão Sistemática e Metanálise de Múltiplos Tratamentos. 2014. Tese de Doutorado. Universidade Federal do Rio Grande do Sul.

27. KISNER, Carolyn; COLBY, Lynn Allen. Exercícios terapêuticos: fundamentos e técnicas. (tradução Lilia Breternitz Ribeiro), 6ª ed. Barueri, SP: Manole, 2016. 